\title{
AVALIAÇÃO DA DESIDRATAÇÃO OSMÓTICA E IMPREGNAÇÃO A VÁCUO ASSOCIADOS COM A SECAGEM CONVECTIVA DE ABACAXI (VAR. ANANAS COMOSUS L.)
}

\author{
P. G. SOUZA ${ }^{1}$, B. A. M. CARCIOFI ${ }^{1}$. \\ ${ }^{1}$ Universidade Federal de Santa Catarina, Departamento de Engenharia Química e Engenharia \\ de Alimentos \\ E-mail para contato: gimenez.paulinha@gmail.com
}

\begin{abstract}
RESUMO: O abacaxi é uma fruta altamente perecível e, por isso, necessita do desenvolvimento de processos para sua preservação. Diversos métodos de desidratação podem ser empregados para essa finalidade de forma a aumentar a vida útil do produto pela redução da sua atividade de água. O objetivo desse trabalho foi comparar diferentes processos de preservação. As frutas foram submetidas a 5 processos: desidratação osmótica (DO), secagem convectiva (SC), DO com SC, impregnação a vácuo (IV) com DO e IV com SC. A DO e a IV foram realizadas em solução de sacarose a $57^{\circ}$ Brix e $45{ }^{\circ} \mathrm{C}$ com proporção fruta:solução de 1:25(m/m), sendo que a DO ocorreu por $48 \mathrm{~h}$. A SC foi conduzida em estufa com circulação forçada de ar a $60{ }^{\circ} \mathrm{C}$ e $70{ }^{\circ} \mathrm{C}$ por $10 \mathrm{~h}$. Na IV as amostras permaneceram imersas na solução por 10 min sob pressão de $100 \mathrm{mmHg}$ e por adicionais $10 \mathrm{~min}$ sob a pressão de $760 \mathrm{mmHg}$. Após o processamento, foram realizadas análises relativas à cinética de redução do teor de água, atividade de água e perda de massa. Nos ensaios de DO e IV foi determinado o ganho de sólidos durante o processo. Constatou-se uma perda de massa de $86 \%$ do valor inicial nos processos de SC e de $12 \%$ no processo de IVDO. A umidade foi reduzida a $4,5 \%$ nos processos de SC, enquanto que na DO$\mathrm{SC}$ de $60{ }^{\circ} \mathrm{C}$ a umidade permaneceu em $35 \%$ do valor correspondente a fruta in natura. O ganho de sólidos demonstrou que a IV corresponde a $1 \mathrm{~h}$ de DO o que possibilita a substituição da DO no enriquecimento com vitaminas e minerais.
\end{abstract}

\section{INTRODUÇÃO}

A produção mundial de frutas tropicais, segundo a Organização das Nações Unidas para Agricultura e Alimentação (FAO), deverá alcançar 82 milhões de toneladas em 2014, sendo que $90 \%$ são produzidas em países em desenvolvimento (ABANORTE, 2012). A América Latina, assim como demais regiões em desenvolvimento, apresentam perdas 
superiores a $40 \%$ relacionadas à produção, pós-colheita, processamento e distribuição de frutas e hortaliças (FAO, 2011). De acordo com a FAO, o Brasil é o maior produtor mundial de abacaxi (FAMASUL, 2014), com perdas estimadas em $24 \%$ (EMBRAPA, 2009). O abacaxi da variedade Pérola é o mais produzido no Brasil, apresentando-se naturalmente com menor acidez em relação à variedade Smooth Cavenne (BENGOZI et al., 2007), a qual, em contraste, encontra-se em destaque para a exportação (ABF, 2013).

Como forma de reduzir as perdas no transporte e distribuição, aumentar a vida útil e agregar valor à fruta é necessário o desenvolvimento de práticas de preservação por meio do processamento do abacaxi Pérola. Com o objetivo de reduzir o teor de água livre nas frutas, diversas práticas podem ser utilizadas, como a secagem convectiva, desidratação osmótica e impregnação a vácuo. Estes processos proporcionam a redução da atividade de água da fruta por meio da retirada parcial de água livre (secagem convectiva) e/ou aumento do teor de sólidos solúveis com a incorporação de uma solução hipertônica de sacarose (desidratação osmótica e impregnação a vácuo). A sacarose é considerada um ótimo agente desidratante pelo fato de evitar reações de escurecimento enzimático (AZOUBEL, 1999) e garantir a preservação do aroma da fruta (RAOULT-WACK, 1994).

Com o intuito de se obter um produto de qualidade superior é possível aplicar os métodos de forma combinada, garantindo que as alterações da matéria-prima sejam realizadas de maneira controlada (TORREGIANNI; BERTOLO, 2001). Neste contexto, o objetivo do presente trabalho foi a aplicação dos métodos de secagem convectiva, desidratação osmótica e impregnação a vácuo de forma individual e combinada com o propósito de verificar quais são as consequências desses processos nos parâmetros físico-químicos do abacaxi desidratado.

\section{MATERIAIS E MÉTODOS}

\subsection{Preparo das Amostras}

Os abacaxis da variedade Pérola (Ananas comosus L.) foram adquiridos no comércio local de Florianópolis - SC. Foram selecionados por meio de análise visual, buscando-se padronizar o grau de maturação das frutas. As frutas foram higienizadas com detergente neutro e descascadas manualmente. Em seguida, foram cortadas fatias da porção central da fruta (descartando-se as extremidades). As fatias tiveram 12,1 $\mathrm{mm}$ de espessura e foram divididas em oitavos de maneira uniforme. Os frutos foram caracterizados pela determinação da concentração de sólidos solúveis, da atividade de água $\left(\mathrm{a}_{\mathrm{w}}\right)$, da umidade e do $\mathrm{pH}$ das amostras in natura.

As amostras utilizadas nos experimentos foram destrutivas com análises em duplicata.

\subsection{Solução Osmótica e Impregnante}

A solução osmótica e impregnante foram preparadas diluindo-se açúcar refinado (sacarose) da marca União com água destilada, a fim de se obter uma concentração de $57^{\circ}$ Brix à $45^{\circ} \mathrm{C}$. A concentração de sólidos solúveis foi determinada com auxílio um de refratômetro digital (Pocket Refractometer, ATAGO®).

\subsection{Desidratação Osmótica (DO)}


O dispositivo experimental utilizado consistiu em uma câmara encamisada com volume interno de 14,14 litros, com controle de temperatura por meio de um banho termostatizado (QUIMIS, Modelo Q214M2) acoplado à camisa do equipamento. No interior da câmara, a solução osmótica foi agitada através de uma bomba submersa de circulação de líquido (Atman, Modelo AT-010) com vazão nominal de 2000 litros por hora. Foi utilizada uma relação mássica fruta:solução de 1:25.

Inicialmente, as amostras foram pesadas e dispostas em telas de nylon previamente identificadas. Em seguida, todas as fatias foram submersas na solução osmótica simultaneamente. As coletas de amostras para análise da cinética do processo ocorreram a cada $15 \mathrm{~min}$ na primeira hora de ensaio, a cada $30 \mathrm{~min}$ na segunda hora e a cada hora nas subsequentes $8 \mathrm{~h}$ de experimento. Após esse período, foram coletadas amostras correspondentes a $24 \mathrm{~h}, 30 \mathrm{~h}$ e $48 \mathrm{~h}$ de DO.

\subsection{Secagem Convectiva (SC)}

Inicialmente, as amostras foram pesadas e dispostas em placas de Petri previamente identificadas. Todas as fatias foram colocadas simultaneamente na estufa com circulação forçada de ar (TECNAL-TE 394/2), com velocidade do ar de secagem cerca de $1 \mathrm{~m} / \mathrm{s}$ medida com um anemômetro térmico compacto. As temperaturas utilizadas foram de $60{ }^{\circ} \mathrm{C}$ e $70^{\circ} \mathrm{C}$.

As medidas da variação da massa das amostras para posterior avaliação da cinética de secagem ocorreram a cada 15 min na primeira hora de ensaio, a cada 30 min na segunda hora e a cada hora nas subsequentes $8 \mathrm{~h}$ de experimento.

\subsection{Desidratação Osmótica com Secagem Convectiva a $60^{\circ} \mathrm{C}(\mathrm{DO}-\mathrm{SC})$}

A DO ocorreu conforme descrito no item 2.3, sendo que o processo foi encerrado com a retirada de todas as amostras simultaneamente após $45 \mathrm{~min}$ de ensaio. Em seguida, as amostras foram submetidas à secagem a $60^{\circ} \mathrm{C}$ como descrito no tópico 2.4. As análises para caracterização das amostras ocorreram após a DO e nos tempos respectivos da secagem.

\subsection{Impregnação a Vácuo (IV) com Desidratação Osmótica (DO)}

O dispositivo experimental utilizado para IV consiste em uma câmara encamisada com volume interno de 58 litros e com controle da temperatura por meio de um banho termostatizado (QUIMIS, Modelo Q214M2) acoplado à camisa do equipamento. No interior da câmara, a solução impregnante foi agitada por uma bomba sanitária (BOMBINOZ, Modelo BL5) com vazão nominal de 2000 litros por hora, sendo esta desligada no momento da aplicação do vácuo.

As amostras foram pesadas e dispostas em telas de nylon previamente identificadas. Em seguida, todas as fatias foram submersas simultaneamente na solução impregnante e realizou-se uma redução na pressão interna da câmara para $100 \mathrm{mmHg}$, a qual foi mantida por $10 \mathrm{~min}$. Em seguida, foi reestabelecida a pressão atmosférica (aproximadamente $760 \mathrm{mmHg}$ ), permanecendo as amostras submersas por adicionais 10 min. Após este procedimento, a bomba sanitária foi religada e realizou-se a DO como descrito no tópico 2.3, na própria câmara de impregnação. 
As análises ocorreram ao final da IV e nos tempos de retirada descritos no procedimento de DO.

\subsection{Impregnação a Vácuo com Secagem Convectiva a $60^{\circ} \mathrm{C}$ (IV-SC)}

A IV foi realizada como descrito no tópico 2.6. e, após o reestabelecimento da pressão atmosférica, realizou-se o procedimento de secagem a $60^{\circ} \mathrm{C}$ descrito no tópico 2.4. As análises ocorreram após a IV e nos intervalos de tempo de retirada para o processo SC e após $24 \mathrm{~h}, 30 \mathrm{~h}$ e $48 \mathrm{~h}$ de ensaio.

\subsection{Análises Pós-Processamento}

Os dados foram tratados de modo a se determinar a cinética de perda de massa, da variação da atividade de água e da diminuição da umidade para os processamentos aplicados, além da cinética do ganho de sólidos para os processos de IV e DO.

Nos experimentos que envolveram a solução osmótica ou impregnante, o excesso de solução de sacarose aderida à superfície das frutas foi eliminado colocando-as em contato com papel filtro por $5 \mathrm{~s}$ em cada lado anteriormente às análises.

Perda de massa - PM (\%): foi calculada em relação à massa da fatia in natura como representado pela Equação 1 .

$$
\% P M=\frac{\left(m_{t}-m_{i}\right)}{m_{i}} * 100
$$

Onde $\mathrm{m}_{\mathrm{i}}$ é a massa inicial e $\mathrm{m}_{\mathrm{t}}$ é a massa no tempo de retirada da amostra.

Atividade de água - $\mathrm{a}_{\underline{\mathrm{w}}}$ : foi determinada por meio de amostras trituradas, com o auxílio de um higrômetro digital (Aqualab Model Series 3, Decagon Devices, Inc. Pullman, USA).

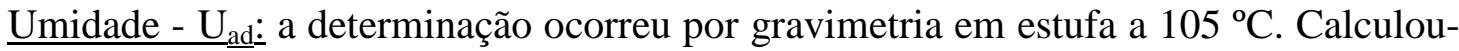
se a umidade adimensional $\left(\mathrm{U}_{\mathrm{ad}}\right)$ utilizando a umidade da amostra in natura como base de cálculo, como representado na Equação 2 para as etapas de DO e IV e a Equação 3 para as etapas de SC. A diferença nas Equações 2 e 3 deve ser considerada, pelo fato de existir o ganho de sólidos nos processos de DO e IV, enquanto que na SC ocorre apenas a saída de água da fruta.

$$
\begin{aligned}
U_{a d} & =\frac{m_{H 2 O}^{t}}{m_{H 2 O}^{i}} \\
U_{a d} & =1-\left(\frac{m_{i}-m_{t}}{m_{i}-m_{s S}}\right)
\end{aligned}
$$

Onde, $\mathrm{m}_{\mathrm{H} 2 \mathrm{O}}^{\mathrm{O}}$ é a massa de água da amostra coletada no tempo $\mathrm{t}, \mathrm{m}_{\mathrm{H} 2 \mathrm{O}}^{\mathrm{i}}$ é a massa de água da amostra in natura, $\mathrm{m}_{\mathrm{i}}$ é a massa inicial da amostra, $\mathrm{m}_{\mathrm{t}}$ é a massa da fruta em cada tempo de retirada e $\mathrm{m}_{\mathrm{ss}}$ é a massa de sólidos secos da fatia. 
Ganho de sólidos - GS: determinou-se o ganho de sólidos adimensional em relação à fruta in natura como representado na Equação 4.

$$
G S=\frac{m_{s S}^{f}-m_{S S}^{i}}{m_{S S}^{i}}
$$

Onde, $\mathrm{m}_{\mathrm{ss}}^{\mathrm{f}}$ é a massa de sólidos secos da amostra, $\mathrm{m}_{\mathrm{ss}}^{\mathrm{i}}$ é a massa de sólidos secos da amostra in natura.

\subsection{Taxa de Secagem Inicial}

Com a finalidade de calcular a taxa de secagem inicial dos processos, foram consideradas as amostras coletadas em tempos inferiores a cinco horas, por se tratar do período com taxa constante de secagem, aproximando, assim, os dados por meio da equação da reta, conforme a Equação 5.

$$
y=a x+b
$$

\section{RESULTADOS E DISCUSSÃO}

$\mathrm{Na}$ secagem convectiva na temperatura de $70^{\circ} \mathrm{C}$ foram observadas alterações indesejáveis (imagens não apresentadas), como um maior encolhimento do tecido vegetal e formação de rachaduras na superfície da fruta. Assim, a SC a $60^{\circ} \mathrm{C}$ foi definida para os demais ensaios combinados pelo fato de se atingir valores de perda de massa e umidade similares ao processo de SC a $70{ }^{\circ} \mathrm{C}$, como demonstrado na Figura 1, sem prejudicar a aparência do produto final.

É possível analisar na Figura 1 que os processos com menor perda de massa percentual foram os de DO e IV-DO, resultado do ganho de sólidos durante as etapas de DO e IV. Em relação aos experimentos envolvendo a DO-SC e IV-SC, percebe-se que houve uma redução maior na massa das amostras submetidas à IV-SC, possivelmente pelo fato de a IV gerar uma alteração na estrutura da fruta pelo fenômeno de deformação-relaxação.

Segundo a Tabela 1 pode-se observar que os pontos relativos ao período de taxa de secagem constante podem ser bem representados pela equação da reta e que houve similaridade entre as taxas de secagem para a SC a $70{ }^{\circ} \mathrm{C}$ e a SC a $60{ }^{\circ} \mathrm{C}$, assim como as taxas entre os processos que foram submetidos a IV (20 min) e a DO (45 min). Isto comprova que a incorporação de sacarose reduz a taxa de secagem, pelo fato de se ligar as moléculas de água livre. 
Tabela 1 - Taxa de secagem inicial das amostras retiradas nas primeiras cinco horas de ensaio para cada processo, aproximadas pela equação da reta

\begin{tabular}{|c|c|c|}
\hline Processo & $\begin{array}{c}\text { Taxa de Secagem } \\
(\mathrm{kg} \mathrm{de} \mathrm{água/h})\end{array}$ & $\mathrm{R}^{2}$ \\
\hline $\mathrm{SC}-70^{\circ} \mathrm{C}$ & 15,674 & 0,9114 \\
\hline $\mathrm{SC}-60^{\circ} \mathrm{C}$ & 14,178 & 0,9517 \\
\hline $\mathrm{IV}-\mathrm{SC}$ & 8,3564 & 0,9174 \\
\hline $\mathrm{IV}-\mathrm{DO}$ & 8,6234 & 0,9648 \\
\hline
\end{tabular}

Na Figura 2 é possível constatar que é necessário realizar a IV e a DO combinadas com a SC para que exista uma redução mais significativa em relação à atividade de água $\left(a_{w}\right)$, de forma a evitar o crescimento de bactérias segundo as taxas generalizadas de reações de deterioração em alimentos em função da atividade de água em temperatura ambiente (VAN DEN BERG; BRUIN, 1981). Além disso, observa-se que os processos combinados DO-SC e IV-SC proporcionam uma $\mathrm{a}_{\mathrm{w}}$ inferior ao processo de $\mathrm{SC}-60^{\circ} \mathrm{C}$ em virtude dos fluxos de retirada de água e incorporação de sólidos que os pré-processamentos impõe a SC, de forma a reduzir o teor de água livre na fruta, aumentando a vida útil do produto.

Ao se analisar a Figura 3 nota-se que a umidade foi reduzida a 4,5\% do seu valor inicial ao final das $10 \mathrm{~h}$ de SC, enquanto que ao se realizar a DO-SC esse parâmetro permaneceu em $35 \%$ do valor correspondente ao da fruta in natura. Observa-se também que a IV reduz a umidade de forma a contribuir para a retirada de água livre nos processos combinados. Nesse caso, os processos de IV-DO e IV-SC apresentaram valores distintos para a umidade ao final do processo de IV, possivelmente pelo fato de se tratarem de amostras de frutas, que naturalmente apresentam variações. No entanto, os valores de umidade da DO e IV-DO permaneceram similares ao final dos respectivos ensaios, pelo fato de existir uma contribuição prolongada da DO sobre as amostras.

Pela análise da Figura 4 observa-se que o ganho de sólidos correspondente ao processo de IV (20 mim) foi igual ao de $1 \mathrm{~h}$ de ensaio de DO. Contudo, apresentou uma similaridade com o decorrer dos processos, atingindo um ganho de 6,5 vezes de sólidos em relação ao valor presente na fruta in natura. Nota-se também que o período de maior ganho de sólidos na DO foi nos primeiros $45 \mathrm{~min}$ de ensaio, motivo pelo qual esse tempo foi definido para se realizar o experimento DO-SC. Assim, quando o interesse for relacionado à incorporação quantitativa de sólidos pode-se realizar a DO sem a necessidade de um pré-tratamento com IV, no entanto, se o objetivo for a incorporação rápida de sólidos com redução da atividade de água torna-se viável a aplicação da IV em detrimento da DO. 
Figura 1 - Perda percentual de massa com o tempo em 10 horas de ensaio.

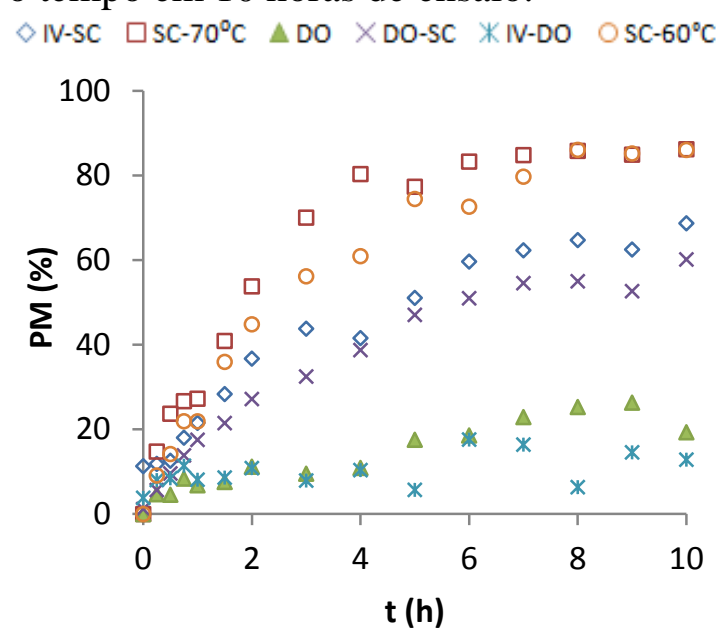

Figura 3 - Variação da umidade com o tempo em 10 horas de ensaio em relação a amostra in natura.

$\diamond \mathrm{IV}-\mathrm{SC} \quad \square \mathrm{SC}-70^{\circ} \mathrm{C} \triangle \mathrm{DO} \times \mathrm{DO}-\mathrm{SC} \quad * \mathrm{IV}-\mathrm{DO} \quad \mathrm{SC}-60^{\circ} \mathrm{C}$

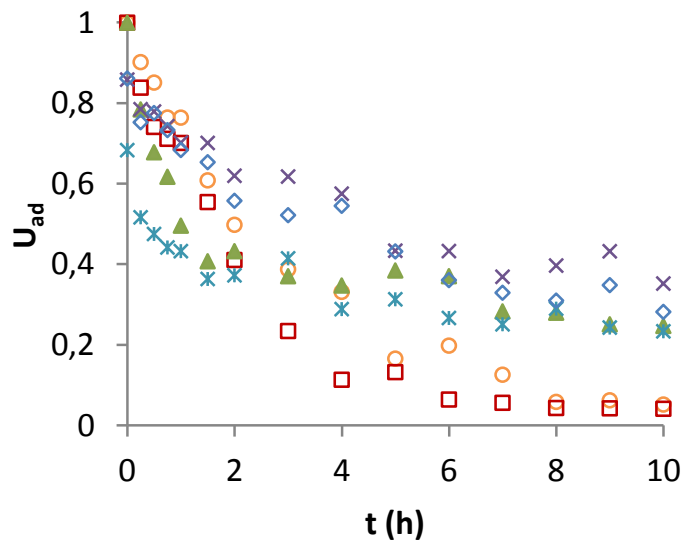

Figura 2 - Variação da atividade de água com o tempo em 10 horas de ensaio.

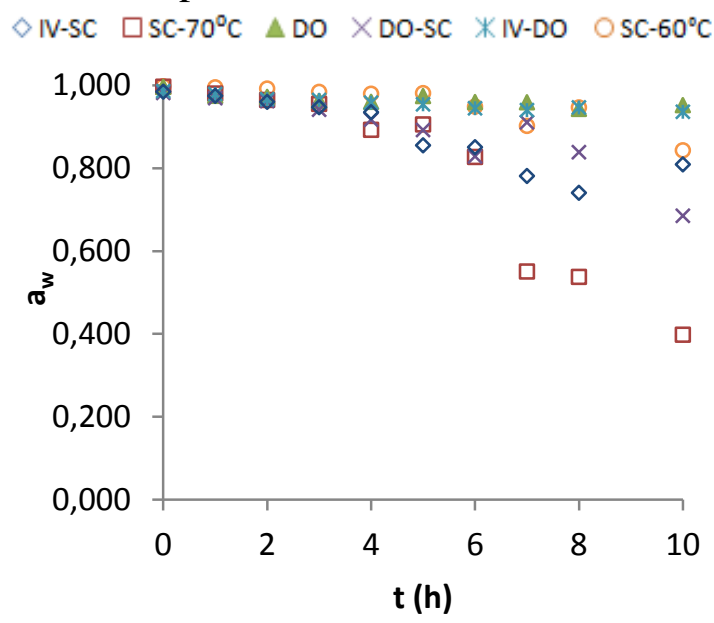

Figura 4 - Ganho de sólidos com o tempo em 48 horas de ensaio em relação a amostra in natura.

$\triangle \mathrm{DO}$ *IV-DO

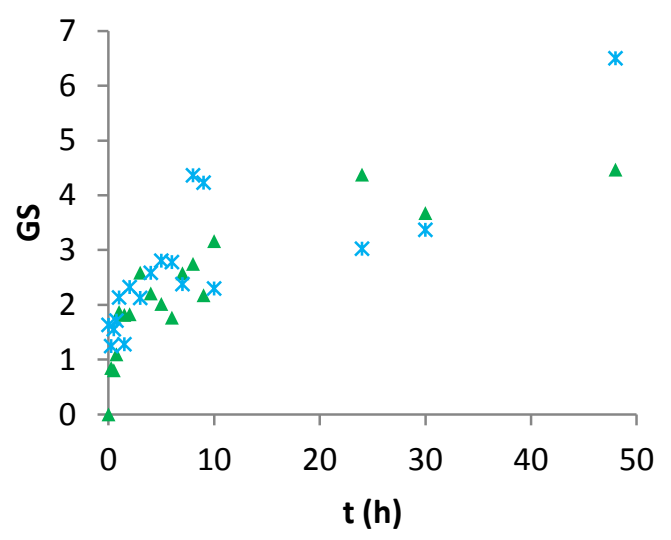

\section{CONCLUSÕES}

Os processos avaliados contribuíram positivamente para a preservação da fruta, por meio da redução da atividade de água e da umidade da amostra, com destaque para os processos de secagem que atingiram valores de $4,5 \%$ de umidade em relação à amostra in natura e 0,045 de atividade de água. Os ensaios que envolveram a incorporação de sacarose garantiram também a redução da atividade de água para valores 0,685 e 0,809 para os processos de DO-SC e IV-SC, com uma diminuição menos drástica da umidade em relação à fruta in natura. Isto demonstra que a utilização de processos combinados de desidratação ou impregnação a vácuo com a secagem convectiva permitem a obtenção de um produto com características mais interessantes para a comercialização com a redução da atividade de água e o aumento do teor de sólidos. 
Além disso, ao se determinar que a IV apresentou um resultado correspondente ao ganho de sólidos de uma hora de DO, pode-se concluir que a IV pode ser utilizada para substituir a DO quando o interesse for relacionado ao ganho de sólidos, desejando-se um processo de menor duração, ou ainda o enriquecimento do produto com vitaminas e minerais.

\section{REFERÊNCIAS}

ANUÁRIO BRASILEIRO DA FRUTICULTURA - ABN. Ed. Gazeta Santa Cruz, Santa Cruz do Sul - RS. 136 p., 2013.

ASSOCIAÇÃO CENTRAL DOS FRUTICULTORES DO NORTE DE MINAS ABANORTE. A produção mundial de frutas tropicais atingirá 82 milhões de toneladas em 2014. Notícias. Portal ABANORTE, out. 2012.

AZOUBEL, P.M. Desidratação osmótica e secagem de tomate cereja (Licopersicum esculetum, var. Cerasiforme). Dissertação (Mestrado). 102 p.1999. FEA, Unicamp, Campinas - SP.

BENGOZI, F. J.; SAMPAIO, A. C.; GUTIERREZ, A. S.; RODRIGUES, V. M.; PALLAMIN, M. L. Análise do mercado do abacaxi comercializado na CEAGESP - São Paulo. Rev. Bras. Frutic., Jaboticabal - SP, v. 29, n.3, p. 494-499, 2007.

FEDERAÇÃO DA AGRICULTURA E PECUÁRIA DE MATO GROSSO DO SUL FAMASUL. Alta produtividade de abacaxi gera renda ao produtor rural. Assessoria de Imprensa Sistema Famasul. Fev., 2014.

INSTITUTO BRASILEIRO DE GEOGRAFIA E ESTATÍSTICA - IBGE. Levant. Sistem. Prod. Agríc. Rio de Janeiro, v. 26, n.1, p. 1-83, 2013.

RAOULT-WACK, A. L. Recent advances in the osmotic dehydration of foods. Food Science \& Tecnology. v. 5, p. 255-260. 1994.

TORREGIANNI, D.; BERTOLO, G. High-quality fruit and vegetables products using combined processes. In FITO, P. et al. (Ed.). Osmotic Dehydration \& Vacuum Impregnation Application in Food Industries. Lancaster: Technomic Publishing Co., Inc., 2001. p. 3-9.

VAN DEN BERG, C.; BRUIN, S. Water activity and its estimation in food systems: theoretical aspects. In: L. B. ROCKLAND; G. F. STEWART (Eds.), Water activity: influences on food quality, New York: Academic Press, p. 1-61, 1981. 